\title{
Is the extrastriate body area part of the dorsal visuomotor stream?
}

\author{
Marius Zimmermann ${ }^{1,2}$ (D) Rogier B. Mars ${ }^{1,3} \cdot$ Floris P. de Lange $^{1} \cdot$ \\ Ivan Toni ${ }^{1}$ (i) $\cdot$ Lennart Verhagen ${ }^{4}$ (it)
}

Received: 18 August 2016/ Accepted: 1 July 2017/Published online: 12 July 2017

(c) The Author(s) 2017. This article is an open access publication

\begin{abstract}
The extrastriate body area (EBA) processes visual information about body parts, and it is considered one among a series of category-specific perceptual modules distributed across the occipito-temporal cortex. However, recent evidence raises the possibility that EBA might also provide an interface between perception and action, linking the ventral and dorsal streams of visual information processing. Here, we assess anatomical evidence supporting this possibility. We localise EBA in individual subjects using a perceptual task and compare the characteristics of its functional and structural connectivity to those of two perceptual areas, the lateral occipital complex (LOC) and the fusiform body area (FBA), separately for each hemisphere. We apply complementary analyses of resting-state fMRI and diffusion-weighted MRI data in a group of healthy right-handed human subjects $(N=31)$. Functional and structural connectivity profiles indicate that EBA interacts more strongly with dorsal-stream regions
\end{abstract}

Electronic supplementary material The online version of this article (doi:10.1007/s00429-017-1469-0) contains supplementary material, which is available to authorized users.

Marius Zimmermann

marius.zimmermann@psychology.su.se

1 Donders Institute for Brain, Cognition and Behaviour, Radboud University, Nijmegen, The Netherlands

2 Department of Psychology, Stockholm University, Stockholm, Sweden

3 Wellcome Centre for Integrative Neuroimaging, Centre for Functional MRI of the Brain (FMRIB), Nuffield Department of Clinical Neurosciences, John Radcliffe Hospital, University of Oxford, Oxford, UK

4 Department of Experimental Psychology, University of Oxford, Oxford, UK compared to other portions of the occipito-temporal cortex involved in processing body parts (FBA) and object identification (LOC). These findings provide anatomical ground for a revision of the functional role of EBA. Building on a number of recent observations, we suggest that EBA contributes to planning goal-directed actions, possibly by specifying a desired postural configuration to parietofrontal areas involved in computing movement parameters.

Keywords Category-selective visual areas · Ventral and dorsal visual pathways - Goal-directed action .

Connectivity profile $\cdot$ Resting-state fMRI $\cdot$ Diffusion MRI

\section{Introduction}

The occipito-temporal cortex extracts information from early visual areas for further perceptual processing along the inferior temporal lobe. This cortical territory is thought to operate as a gateway for perceptual processing in the ventral visual stream and to remain largely separated from a dorsal visual stream that processes visual information relevant to motor control (Goodale and Milner 1992; but see Milner 2017). The extrastriate body area (EBA) has been regarded a case in point. This area responds selectively to images of the human body (Downing et al. 2001). It has been described as a purely perceptual area (Downing and Peelen 2011), processing visual information in a fashion similar to other category-specific regions in ventral occipito-temporal cortex such as the fusiform face area (FFA) (Kanwisher et al. 1997; Hutchison et al. 2014).

However, it has been suggested that EBA might also provide an interface between perceptual and motor processes (Astafiev et al. 2004; David et al. 2007; Gallivan et al. 2011; Kühn et al. 2011; Bracci et al. 2012; Tomasino 
et al. 2012; Limanowski et al. 2014; Orgs et al. 2016; Simos et al. 2017). Such an interface is required during goal-oriented behaviour that relies on perceptual knowledge, as when grasping a hammer according to its use. EBA could interface perceptual and motor processes in two ways. One possibility is that EBA's contributions resemble that of the lateral occipital complex (LOC) (Malach et al. 1995). LOC provides access to perceptual information of object representations (James et al. 2003; Verhagen et al. 2008; Gallivan et al. 2015), and EBA might implement the same perceptual function for representations of body parts (Pitcher et al. 2009; Hutchison et al. 2014; Lingnau and Downing 2015). Another possibility is that EBA is more directly involved in motor control, specifying a desired postural configuration chosen from multiple possibilities during object manipulating actions (van Nuenen et al. 2012; Zimmermann et al. 2012, 2016). Here, in a strongly hypothesis-driven approach, we investigate the anatomical evidence to distinguish between the patterns of connectivity implied by those two possibilities.

Building on recent explorative whole-brain analyses of occipito-temporal connectivity at rest (Hutchison et al. 2014; Lingnau and Downing 2015) and investigations of EBA activity and connectivity in the context of task-related networks (e.g., Beer et al. 2013; Zimmermann et al. 2013; Orgs et al. 2016; Simos et al. 2017), we test whether EBA shows stronger functional and structural connectivity to the dorsal visuomotor stream than other areas of the occipitotemporal cortex that are sensitive to stimulus category. If the role of EBA is mainly perceptual, then this area is expected to have a connectivity profile similar to those of other portions of the ventral visual stream involved in processing body parts (i.e., the fusiform body area, FBA; Peelen and Downing 2005) and in identifying objects (i.e., the lateral occipital complex, LOC; Malach et al. 1995). If EBA directly contributes to planning goal-directed actions, then the connectional affinity of this area with dorsalstream regions is expected to be stronger than that of either FBA or LOC. We distinguish between these two possibilities by considering two complementary indexes of connectivity, diffusion-weighted MRI (dw-MRI) and restingstate fMRI (rs-fMRI). Dw-MRI is a structural index of anatomical connectivity, ideally suited for non-invasive mapping of white-matter fibre systems, whereas restingstate fMRI is a functional index of (multi-synaptic) anatomical connectivity (O'Reilly et al. 2013), based on intrinsic coupled modulations in spontaneous activation between brain areas in the absence of external stimuli or task demands (Biswal et al. 1995; Fox and Raichle 2007; Hagmann et al. 2008; Honey et al. 2009). The strength and novelty of this study lie in combining data-driven and hypothesis-driven analyses of both functional and structural connectivities to make statements about EBA's position within dorsal and ventral visual stream circuits (Passingham 2013).

\section{Methods}

\section{Overview}

The data used in this study were collected at the onset of a larger multi-session study (Zimmermann et al. 2016), but they have not been reported before.

\section{Participants}

Thirty-two healthy, right-handed participants ( $25 \pm 3$ years, 17 male) gave written informed consent to take part in the study and were financially compensated at a rate of $10 \mathrm{euro} / \mathrm{h}$. One subject was excluded from the resting-state analyses due to excessive head movements (>3 mm) during rs-fMRI data collection.

\section{MR scans and procedures}

Each participant completed a series of four scans. All scans were completed within one session in a $1.5 \mathrm{~T}$ MR scanner (Avanto; Siemens, Erlangen, Germany) equipped with a 32-channel head coil for signal reception. Following an anatomical scan (T1-weighted MP-RAGE sequence, TR/ $\mathrm{TE}=2300 / 3.03 \mathrm{~ms}$, voxel size $1 \times 1 \times 1 \mathrm{~mm}$ ), participants performed a visual 1-back task that served as functional localizer for EBA, FBA, and LOC (see "Acquisition of functional localizer scans for EBA, FBA and LOC"), followed by resting-state and diffusion-weighted MRI scans (see "Acquisition of resting-state fMRI and diffusion-weighted MRI data").

During all scans, subjects lay in supine position in the scanner. Cushions on each side of the head were used for stabilization. In addition, subtle tactile feedback about head movements was provided to the subjects by spanning tape from both sides of the head coil over the forehead, making it easier for them to minimize movements. A mirror construction attached to the head coil allowed participants to see a screen at the head end of the scanner bore, where stimuli could be presented.

\section{Acquisition of functional localizer scans for EBA, FBA, and LOC}

Functional localization of EBA, FBA, and LOC was done using a 1-back task to enforce attention to the stimuli. Three sets of stimuli were used during this task. For localization of EBA and FBA, a set of 20 pictures of human bodies with digitally occluded heads was used; for 
LOC, we used a set of 20 pictures of man-made objects (e.g., keyboard, guitar, and window); the third set consisted of phase-scrambled versions of the 20 object pictures. Stimuli were presented in blocks of 20 stimuli +2 stimuli repetitions that had to be detected by the participants (1back task; 10 blocks per condition). Stimulus presentation time was $300 \mathrm{~ms}$ with a $450 \mathrm{~ms}$ inter-stimulus interval. Across trials, the location of the stimuli on the screen was randomly shifted (stimulus size: $\sim 10^{\circ}$ visual angle, shifted by $3.5^{\circ}$ horizontally/vertically). Participants held a button box in their right hand and used their index finger to press a single button on the response box.

For the localizer scan, we acquired 256 whole-brain $\mathrm{T} 2 *$-weighted multi-echo planar images $[\mathrm{TR}=2180 \mathrm{~ms}$, $\mathrm{TE}(1)=9.4 \mathrm{~ms}, \quad \mathrm{TE}(2)=21.2 \mathrm{~ms}, \quad \mathrm{TE}(3)=33.0 \mathrm{~ms}$, $\mathrm{TE}(4)=45.0 \mathrm{~ms} ; 31$ slices, voxel-size $3.5 \times 3.5 \times 3.0$ $\mathrm{mm}$, gap-size $0.5 \mathrm{~mm}]$.

\section{Acquisition of resting-state fMRI and diffusion- weighted MRI data}

Participants were instructed to keep awake with their eyes closed for the time of the resting-state scan, which lasted about $10 \mathrm{~min}$. The dw-MRI scan took $9 \mathrm{~min}$. The light in the scanner room was dimmed during both scans.

The resting-state scan consisted of 266 whole-brain T2*-weighted multi-echo planar images $[\mathrm{TR}=2000 \mathrm{~ms}$, $\mathrm{TE}(1)=6.9 \mathrm{~ms}, \quad \mathrm{TE}(2)=16.2 \mathrm{~ms}, \quad \mathrm{TE}(3)=25.0 \mathrm{~ms}$, $\mathrm{TE}(4)=35 \mathrm{~ms}, \mathrm{TE}(5)=44 \mathrm{~ms} ; 39$ slices, voxel-size $3.5 \times 3.5 \times 3.0 \mathrm{~mm}$, gap-size $0.5 \mathrm{~mm}$ ]. Diffusion-weighted data were acquired using echo planar imaging (64 $2.2 \mathrm{~mm}$-thick axial slices; field of view $220 \times 220 \mathrm{~mm}$; voxel size $2.2 \times 2.2 \times 2.2 \mathrm{~mm}$ ). Diffusion weighting was isotropically distributed along 61 directions using a $b$ value of $1000 \mathrm{~s} / \mathrm{mm}^{2}$. Seven volumes with no diffusion weighting were acquired throughout the acquisition.

\section{Image preprocessing and analysis of functional MRI data}

All functional images, for the localizer task as well as the resting-state scan, were analysed using MATLAB (R2009b; MathWorks, Natick, MA, USA) and SPM8 (Wellcome Department of Cognitive Neurology, London, UK). Regions of interest (ROI) masks were constructed using MarsBaR (Brett et al. 2002).

First, functional images were spatially realigned using a least-squares approach that estimates rigid-body transformations (translations and rotations) by minimizing head movements between the first echo of each image and the reference image (Friston et al. 1995a). Next, all echoes of one image were combined into a single volume. For this, the first 30 volumes of each timeseries (functional localizer scan or resting-state scan) were used to estimate the best weighted echo combination to optimally capture the BOLD response over the brain (Poser et al. 2006). These weights were then applied to the entire timeseries. Subsequently, the timeseries for each voxel were temporally realigned to the acquisition of the first slice. Anatomical images were spatially coregistered to the means of the functional images. Normalization parameters to transform anatomical images to a standard EPI template centred in MNI space (Ashburner and Friston 1999) were estimated and used to transform individual structural and functional images into a standard space for group analyses, with a voxel size of $2 \times 2 \times 2 \mathrm{~mm}$. Finally, images were smoothed with a $6 \mathrm{~mm}$ full-width at half-maximum (FWHM) kernel.

For the localizer task, square-wave functions corresponding to the block duration were constructed for each of the three image categories (bodies, objects, and scrambles), and convolved with a canonical haemodynamic response function and its temporal derivative (Friston et al. 1995b). In addition, the statistical model included 13 separate regressors of no interest, modelling button presses, and residual head movement-related effects by including the six rigid-body motion parameters (translations and rotations), as well as their first-order temporal derivatives. Parameter estimates for all regressors were obtained by maximum-likelihood estimation, using a temporal highpass filter (cutoff $128 \mathrm{~s}$ ), modelling temporal autocorrelation as a first-order autoregressive process. Linear contrasts pertaining to the main effects of the design were calculated.

EBA and FBA were identified by comparing statistical parametric maps of the 'body' condition with those of the 'object' condition, providing locations for left and right EBA and FBA (Downing et al. 2001; Peelen and Downing 2005). LOC was identified by comparing statistical parametric maps of the 'object' condition with those of the 'scrambled' condition, providing locations for left and right LOC (Malach et al. 1995). For each ROI, we identified the most significantly activated voxel within a restricted area of the cortex, which was based on previously published locations (EBA: Downing et al. 2001; FBA: Peelen and Downing 2005; LOC: Malach et al. 1995).

Using individual locations for left and right EBA, FBA, and LOC, timeseries for the seed regions were extracted from the resting-state data for each participant. Separate GLMs were constructed for each of the six seed regions (1/ rEBA, 1/rFBA, and 1/rLOC). Each GLM included the first eigenvalue timeseries of a $4 \mathrm{~mm}$ sphere around the individuals' peak coordinates for the seed region. 15 additional regressors were included in each design matrix, modelling residual head movement effects by including the six rigidbody motion parameters (translations and rotations), as well as their first temporal derivatives, and compartment signals for white matter, cerebro-spinal fluid, and out-of- 
brain signals (Verhagen et al. 2008). Parameter estimates for the connectivity between the seed region and the rest of the brain were obtained by maximum-likelihood estimation, using a temporal high-pass filter (cutoff $128 \mathrm{~s}$ ) and modelling temporal autocorrelation as a first-order autoregressive process. Estimated beta-maps related to the seed regions' time-course regressors were used for subsequent analyses (see below).

\section{Functional connectivity analyses: overview}

First, we characterized the connectivity patterns for the different seed regions EBA, FBA, and LOC in a descriptive analysis to identify connected regions at the whole-brain level ("Part I: explorative analyses"). Differences and similarities between these patterns were illustrated using connectivity fingerprints. Subsequently, we quantified differences between these patterns in two complementary statistical analyses ("Part II: hypothesis-driven analyses"). Differences in connectivity strength with dorsal and ventral stream regions were quantified using ROI-based analyses ("ROI-based functional connectivity"). Differences between connectivity patterns of EBA, FBA, and LOC were quantified using a multivariate classifier trained on whole-brain connectivity patterns of the superior parietal and inferior temporal lobules ("Seed-region classification on whole-brain connectivity patterns").

\section{Part I: explorative analyses}

Estimating whole-brain connectivity patterns and contrasts To describe the connectivity pattern of EBA, FBA, and LOC, we identified areas whose timeseries were correlated with those of the seed regions at the whole-brain level. In SPM, we conducted a within-subject analysis of the beta-images obtained during the first-level analysis (see "Image preprocessing and analysis of functional MRI data"), treating participants as a random factor. In addition, we contrasted the connectivity patterns to identify the areas that are differently co-activated with the seed regions, in both directions (e.g., EBA > LOC, LOC > EBA). Both whole-brain analyses were performed using a two-step procedure, where first clusters were formed using a clusterforming threshold of $p<0.001$ (uncorrected), followed by identification of significant clusters at $p<0.05$ (FWE corrected). Where possible, activated clusters were assigned an anatomical label using SPM's Anatomy toolbox (Eickhoff et al. 2005).

Creation of connectivity fingerprints To further describe connectivity profiles for the seed regions, we established 'fingerprints' based on coupling with a set of 13 ipsilateral target ROIs in MNI space (Table 1). The purpose of the
Table 1 List of target regions and MNI coordinates used for connectivity fingerprints (Fig. 4)

\begin{tabular}{lllll}
\hline Label & Region & MNI & & \\
\cline { 3 - 5 } & & $X$ & $Y$ & $Z$ \\
\hline BA45 & Brodmann area 45 & \pm 54 & +24 & +16 \\
BA2 & Brodmann area 2 & \pm 42 & -30 & +48 \\
OP & Parietal operculum & \pm 54 & -18 & +18 \\
BA5 & Brodmann area 5 & \pm 18 & -52 & +62 \\
BA7a & Brodmann area 7 (anterior) & \pm 32 & -43 & +58 \\
V1 & Primary visual cortex & \pm 8 & -90 & +2 \\
FFG & Fusiform gyrus & \pm 42 & -50 & -8 \\
IT & Inferior temporal lobe & \pm 35 & -36 & -20 \\
MTS & Medial temporal sulcus & \pm 58 & -36 & -4 \\
STS & Superior temporal sulcus & \pm 58 & -28 & +14 \\
Ins & Insula & \pm 52 & +4 & -14 \\
Hipp & Hippocampus & \pm 20 & -34 & -4 \\
OFC & Orbito-frontal cortex & \pm 4 & +58 & -14 \\
\hline
\end{tabular}

fingerprints was to simplify visualisation of the wholebrain connectivity maps, i.e., the similarities and differences between the maps of the different seed regions. The target regions were a relevant sample selected a posteriori from the whole-brain connectivity maps and contrast maps between seed regions, choosing regions that would add most value to visualize similarities and differences between connectivity profiles. Regions were centred on peaks in the statistical parametric maps. Each target region consisted of a sphere with $4 \mathrm{~mm}$ radius, equivalent to 33 voxels, created with MarsBaR and SPM8. Per subject and localizer ROI seed region, average beta values of the target ROIs were extracted and summarized over participants. Values were masked at zero, following procedures used by Mars and colleagues (2011) (Sallet et al. 2013; Neubert et al. 2014). Spider-plot diagrams were created, illustrating the connectivity fingerprint of each seed region.

We quantified the similarities and differences between fingerprints using connectivity fingerprint matching (Mars et al. 2016b). Permutation testing (Nichols and Holmes 2002) was used to test the significance of the difference between seed regions within each hemisphere and between homologues across hemispheres. We tested the hypothesis that the difference between regions' connectivity fingerprints, as indexed by the city-block distance (i.e., the sum of differences over all fingerprint arms between a pair of regions), is larger than expected by chance. To obtain a robust estimate of the chance level, we calculated the cityblock distance for each relevant pair of fingerprints for 5000 different permutations of the seed-region labels. For each test, the fingerprints were normalized to a range between 0 (weakest connection with any of the target regions) and 1 (strongest connection with any of the target 
regions). In order not to bias the test, the normalization was performed over the combined arms of both fingerprints. Subsequently, we used logistic regression to assess which target regions significantly contributed to observed differences between hemispheric homologues. Given that these analyses served to further illustrate the whole-brain analyses, these statistical analyses were not corrected for multiple comparisons.

\section{Part II: hypothesis-driven analyses}

Definition of dorsal and ventral stream target regions In the following analyses, we investigate the connectivity between the seed regions and regions of interest representing the core of the dorsal and ventral stream. Following Goodale and Milner (1992), Milner and Goodale (2008), for the purposes of the current study, we focus on the 'dorsal vision-for-action' stream along the lateral superior and inferior parietal lobes, 'dorsal stream' in short. Similarly, we focus on the 'ventral vision-for-identification' processing stream along the inferior temporal lobe, 'ventral stream' in short. Dorsal and ventral stream ROIs were based on sets of the cortical parcellation of Glasser and colleagues using multi-modal analyses of magnetic resonance images from the Human Connectome Project (HCPMMP1.0 atlas, Glasser et al. 2016). The choice of regions that constitute our dorsal and ventral stream regions of interest was based on a review of existing literature on the two streams (Mishkin and Ungerleider 1982; Felleman and Van Essen 1991; Goodale and Milner 1992; Young 1992; Milner and Goodale 2008; Kravitz et al. 2011, 2013) and on a description of the HCP-MMP1.0 atlas (Glasser et al. 2016). In short, we have aimed to select regions with a consistent functional profile adhering to our hypothesis while being both generously inclusive to avoid selection bias and restrictive, where proximity bias might otherwise potentially skew the results. Specifically, for the dorsalstream region of interest, we included lateral regions of the posterior parietal cortex, spanning both the superior and inferior parietal lobe (avoiding selection bias). We excluded occipital areas to adhere to our hypothesis focussed on 'vision-for-action' (Young 1992) and to avoid potentially artificially strong connectivity of EBA with our target ROI due to proximity. Similarly, we excluded parietal regions that constituted clearly distinct functional processing circuits with characteristic connectional profiles, both along the medial wall (Kravitz et al. 2011) and belonging to the temporoparietal junction (TPJ; Mars et al. 2011). For the ventral stream region of interest, we focussed on the whole inferior temporal lobe while excluding several bordering areas following the same criteria as for the dorsal-stream definition. Namely, we excluded occipital regions to avoid proximity bias, and areas in lateral and superior temporal
Table 2 List of regions selected from the HCP-MMP1.0 atlas (Glasser et al. 2016) forming part of dorsal and ventral stream ROIs

\begin{tabular}{|c|c|c|}
\hline Area name ${ }^{a}$ & Area description $^{\mathrm{a}}$ & Stream \\
\hline TE1a & Area TE1 anterior & Ventral \\
\hline TE1p & Area TE1 posterior & Ventral \\
\hline TE2a & Area TE2 anterior & Ventral \\
\hline $\mathrm{TF}$ & Area TF & Ventral \\
\hline TE2p & Area TE2 posterior & Ventral \\
\hline PHT & Area PHT & Ventral \\
\hline $\mathrm{PH}$ & Area PH & Ventral \\
\hline TGv & Area TG ventral & Ventral \\
\hline TE1 m & Area TE1 middle & Ventral \\
\hline IPS1 & Intraparietal sulcus area 1 & Dorsal \\
\hline $7 \mathrm{AL}$ & Lateral area $7 \mathrm{~A}$ & Dorsal \\
\hline 7PI & Lateral area $7 \mathrm{P}$ & Dorsal \\
\hline $7 \mathrm{PC}$ & Area 7PC & Dorsal \\
\hline LIPv & Area lateral intraparietal ventral & Dorsal \\
\hline VIP & Ventral intraparietal complex & Dorsal \\
\hline MIP & Medial intraparietal complex & Dorsal \\
\hline $\mathrm{PFt}$ & Area PFt & Dorsal \\
\hline IP2 & Area intraparietal 2 & Dorsal \\
\hline IP1 & Area intraparietal 1 & Dorsal \\
\hline PF & Area PF complex & Dorsal \\
\hline PFm & Area PFm complex & Dorsal \\
\hline V6A & Area V6A & Dorsal \\
\hline
\end{tabular}

a Area name and area description referring to labels as used in Glasser et al. (2016)

cortex based on their distinctive functional and connectional profiles (including connections with the dorsal stream; Kravitz et al. 2013). The selected regions are listed in Table 2. To obtain individuated regions of interest, we processed each participant's T1-weighted anatomical image according to the open-source HCP Minimal Processing Pipeline (Glasser et al. 2013), or more specifically, according to a version of the Pipeline that does not require a T2-weighted image (https://github.com/lennartverhagen/ Pipelines). This allowed us to directly map the HCPMMP1.0 atlas from the average cortical surface back to a participant's individual volumetric MR image for further statistical analysis.

ROI-based functional connectivity We were interested in assessing the connectivity profile of EBA in relation to those of prototypical perceptual regions such as FBA and LOC. This was tested by investigating the connectivity of the seed regions (EBA, FBA, and LOC) to sets of dorsal and ventral stream regions (see "Definition of dorsal and ventral stream target-regions").

Using Matlab, we extracted the average connectivity strength (beta values) of the seed regions (EBA and LOC) 
with the two ipsilateral target regions (dorsal and ventral). Two-way ANOVAs with factors seed region (EBA, FBA, and LOC) and target region (dorsal and ventral) were used to test for significant interactions between seed- and target regions within each hemisphere. A three-way ANOVA with the additional factor hemisphere (left and right) was used to test for potential differences in hemispheric specialization. For all ROI-based analyses, values of $p<0.05$ were considered statistically significant. In addition, we analysed the connectivity strength of the seed regions with each region of the HCP-MMP1.0 atlas (Glasser et al. 2016) individually. The aim of this analysis was to investigate whether any differences in connectivity strength with dorsal and ventral stream target regions are consistent over a wider range of regions and, therefore, robust against minor changes of our definition of dorsal and ventral stream target regions. The results of this analysis are presented in the supplementary material (Figure S3).

Seed-region classification on whole-brain connectivity patterns Complementary to the above assessment of connectivity strength, we aimed to classify the connectivity patterns of the seed regions (EBA, FBA, and LOC) as resembling either a 'dorsal' or a 'ventral' profile. First, for each of the parcellation areas that together form our dorsal and ventral stream ROIs (see "Definition of dorsal and ventral stream target-regions"; Table 2), we calculated the whole-brain connectivity pattern in SPM8, using the same procedure as we used for the connectivity maps of the seed regions EBA, LOC, and FBA. The statistical parametric maps (beta-images) were labelled as dorsal or ventral according to Table 2, and formed the training set for a $k$ nearest-neighbour classifier in Matab (knnclassify; distance metric: city block). The number of nearest neighbours (k) was modulated from 2 to 8 (i.e., 1 less than the number of sub-regions in the ventral stream). This classifier was then used to classify the connectivity maps (beta-images) of the three seed regions (EBA, LOC, and FBA) for each participant. The outcome of the seed-regions' classification was compared using a Friedman's test with factors seed regions (EBA, LOC, and FBA) and $k$ [2...8]. Friedman's test is a non-parametric version of a balanced two-way ANOVA, which allows for testing of within-subject differences. A three-way ANOVA with the additional factor hemisphere (left and right) was used to test for potential differences in hemispheric specialization. For classification analyses, values of $p<0.05$ were considered statistically significant.

Image processing and analysis of diffusion-weighted MRI Analyses of diffusion-weighted images were performed using tools from FDT v2.0 (FMRIB's Diffusion Toolbox) as part of FSL v5.0 (FMRIB's Software Library) and custom-made software written in Matlab. Eddy-current distortions were corrected using affine registration of all volumes to a target volume with no diffusion weighting. Voxelwise estimates of the fibre orientation distribution were calculated using BedpostX, limited to estimating two fibre orientations at each voxel, because of the $b$ value and number of gradient orientations in the diffusion data (Behrens et al. 2007).

For each participant, the T1 image was linearly registered to the diffusion images (based on an image with no diffusion weighting as a target) using FLIRT (FMRIB's Linear Image Registration Tool) and segmented using FAST (FMRIB's Automated Segmentation Tool). The T1 image was non-linearly warped to the standard MNI space (as defined by the MNI152 template brain provided by FSL) using FNIRT (FMRIB's Non-Linear Image Registration Tool). The resulting transformations were used to register the individual locations for EBA, FBA, and LOC to each participant's diffusion space. Probabilistic tractography was run from all voxels in the sheet of whitematter voxels bordering the grey matter of our regions of interest (as obtained from the HCP Minimal Processing Pipeline). We seeded from the 20 white-matter voxels on the border with grey matter closest to each participant's EBA, FBA, and LOC locations. We included only paths that terminated in the white-matter bordering the dorsal and ventral masks (see "Definition of dorsal and ventral stream target-regions"). We operationalized anatomical connectivity strength as the average of the log-transformed and normalized number of paths that originate from either EBA, FBA, or LOC and reach the dorsal and ventral target regions (Mars et al. 2016a). For analyses of diffusionweighted MRI data, values of $p<0.05$ were considered statistically significant. In addition, we analysed anatomical connectivity strength of the seed regions with each region of the HCP-MMP1.0 atlas (Glasser et al. 2016) individually. Again, the aim of this analysis was to investigate whether any differences in anatomical connectivity with dorsal and ventral stream target regions are consistent over a wider range of regions and, therefore, robust against minor changes of our definition of dorsal and ventral stream target regions. The results of this analysis are presented in the supplementary material (Figure S4).

\section{Results}

\section{Seed regions EBA, FBA, and LOC}

Average MNI coordinates for left and right EBA were $\left[\begin{array}{ll}-49 & -76 \\ 8\end{array}\right]$ and [50 -73 4], for FBA [ $\left.-41-47-18\right]$ and [42 -50 -17], and for LOC [ $-44-80-7]$ and [45 -79 -7]. The average distances between regions differed [left: 


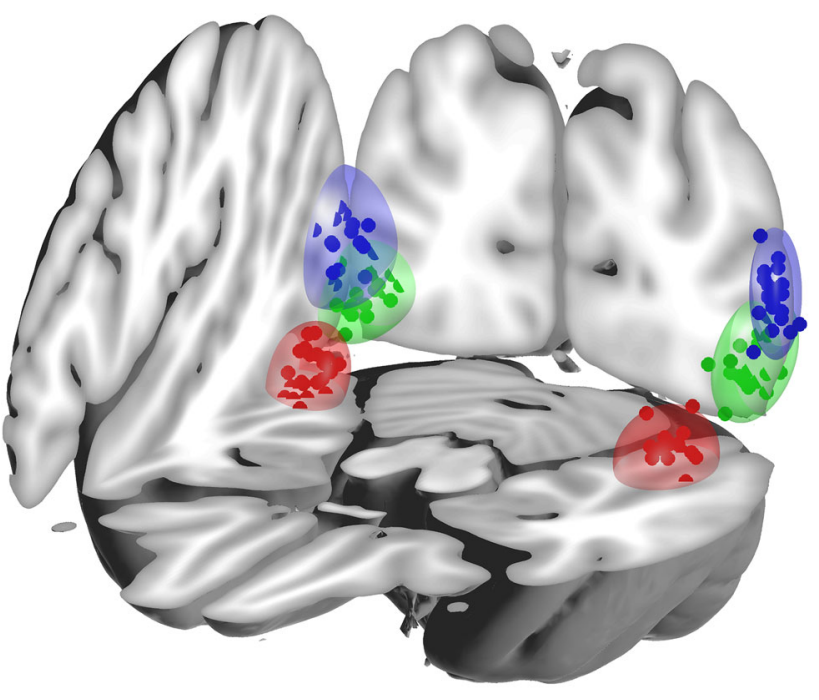

BODIES > OBJECTS

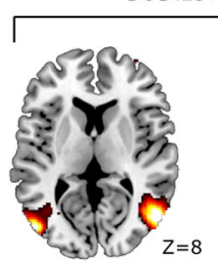

EBA

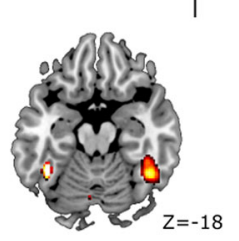

FBA
OBJECTS > SCRAMBLED

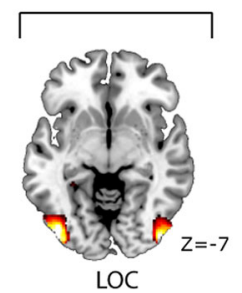

Fig. 1 Top locations of subject-specific seed regions in EBA (blue), LOC (green), and FBA (red). Ellipsoids represent 95\% confidence intervals in all three directions of each ROI location. A control analysis confirmed that there was no overlap between seed regions for any of the participants. Bottom seed-region locations and contrasts for functional localizer EBA, FBA (bodies $>$ objects, $p_{(\mathrm{FWE})}<0.05$; Downing et al. 2001; Peelen and Downing 2005) and LOC (objects > scrambled; Malach et al. 1995)

$F(2,92)=172.94, \quad p<0.001 ; \quad$ right: $\quad F(2,92)=77.9$, $p<0.001]$; the distance between EBA and LOC was on average $17.9 \pm 4.1 \mathrm{~mm}$ (left) and $17.1 \pm 4.7 \mathrm{~mm}$ (right), between EBA and FBA, it was $41.5 \pm 5.1 \mathrm{~mm}$ (left) and $33.4 \pm 6.6 \mathrm{~mm}$ (right), and between LOC and FBA, it was $34.9 \pm 6.3 \mathrm{~mm}$ (left) and $31.6 \pm 5.5 \mathrm{~mm}$ (right) (Fig. 1). A control analysis confirmed that there was no overlap $(0$ voxels) between the seed regions (EBA, FBA, and LOC) for any of the participants.

\section{Part I: explorative analyses}

Whole-brain connectivity patterns We used multiple regression analysis to identify brain regions where BOLD fluctuations were uniquely coupled to those of EBA, FBA, or LOC (Fig. 2). EBA and LOC functional connectivity maps showed large similarities, each sharing unique fluctuations with the occipital cortices, superior temporal lobes, superior parietal lobes, and post- and pre-central regions. FBA was coupled with the inferior temporal and
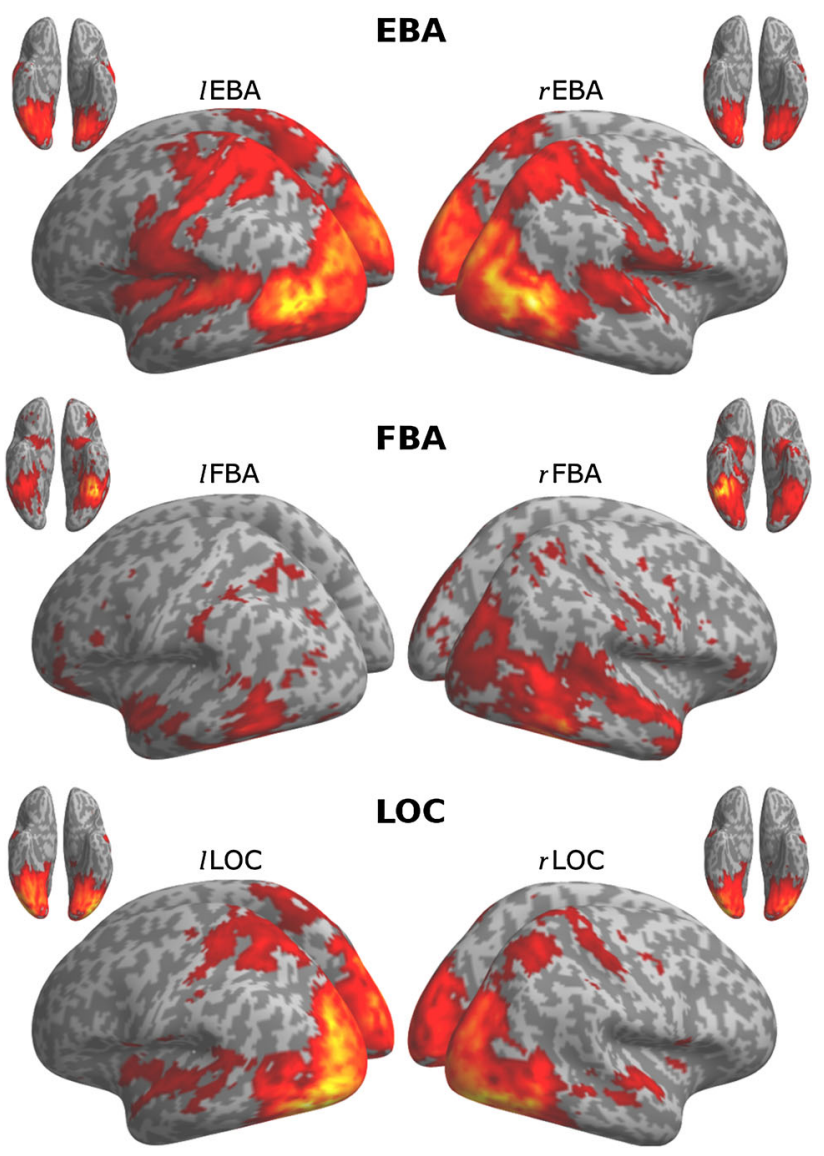

Fig. 2 Whole-brain connectivity patterns for EBA (top), FBA (middle), and LOC (bottom), and left and right hemisphere seed regions. Clusters larger than 50 contiguous voxels are shown, on the basis of a cluster-forming threshold of $p<0.001$. Colours and colour bar (red-yellow) indicate voxelwise $t$ values

occipital lobe and parts of the parietal cortex. These descriptive results replicate earlier findings (Hutchison et al. 2014), opening the way for a novel quantitative test of differences in the connectivity profiles of EBA, FBA, and LOC (Figs. 4, 5). As shown in Fig. 3, and fully reported in the supplementary materials (Table S1), we observed stronger interactions of EBA, compared to LOC, with left and right parietal operculum as well as parts of the midsuperior temporal gyrus. LOC interacts more strongly with areas around the posterior fusiform gyrus and inferior occipital and temporal cortex. EBA, compared to FBA, interacts more strongly with mid-superior temporal and occipital cortices, as well as postcentral regions. EBA interacts more strongly with the pre-central gyrus than either LOC or FBA. FBA, on the other hand, has stronger interactions with the inferior temporal gyrus, fusiform gyrus, parahippocampal gyrus, and pre-SMA. FBA, compared with LOC, interacts more strongly with inferior occipito-temporal cortex, anterior fusiform gyrus, and the 

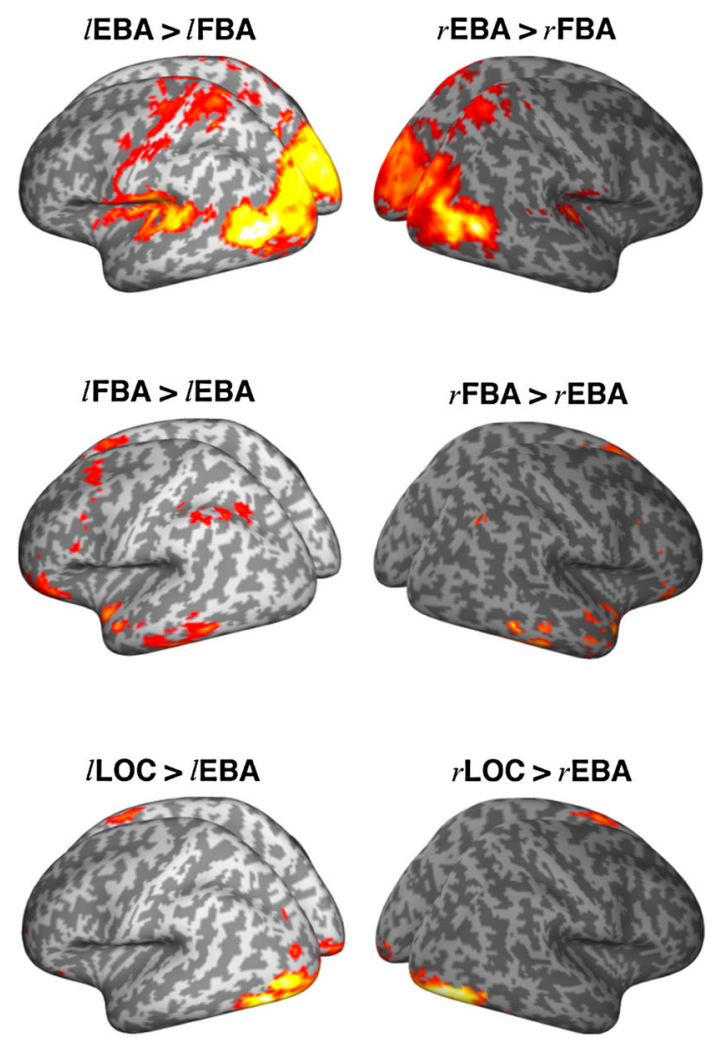

Fig. 3 Contrast images of whole-brain connectivity patterns for left and right seed regions between EBA, FBA, and LOC. Clusters larger than 50 contiguous voxels are shown, on the basis of a cluster-

left hippocampal area. LOC has stronger interactions with large parts of the occipital cortex, compared to FBA. In this exploratory analysis, we did not observe hemispheric specialization in the differences between connectivity patterns of seed regions (Fig. 3).

Connectivity fingerprints To further characterize the connectivity patterns of EBA, FBA, and LOC, we represented the connectivity fingerprints in relation to a set of target regions in a spider plot (Fig. 4) that highlights differences in connectivity fingerprints between EBA, FBA, and LOC, and between left and right homologues. Permutation testing confirmed the exploratory whole-brain results and revealed that each region has an identifiable connectivity fingerprint (all comparisons between pairs of fingerprints $p<0.05$, except rLOC-rFBA: $p=0.06$; uncorrected). These fingerprints could potentially be used to identify these regions based on resting-state fMRI data alone, in the absence of localizer tasks (Saygin et al. 2012; Mars et al. 2013; Tavor et al. 2016; see also Osher et al. 2016). EBA is characterized by connections with the superior temporal sulcus and with parietal regions involved in motor control, including BA5 (SPL), BA7a (anterior IPS), OP, and BA2, which are weaker for the other seed regions. The connectivity fingerprint for FBA is
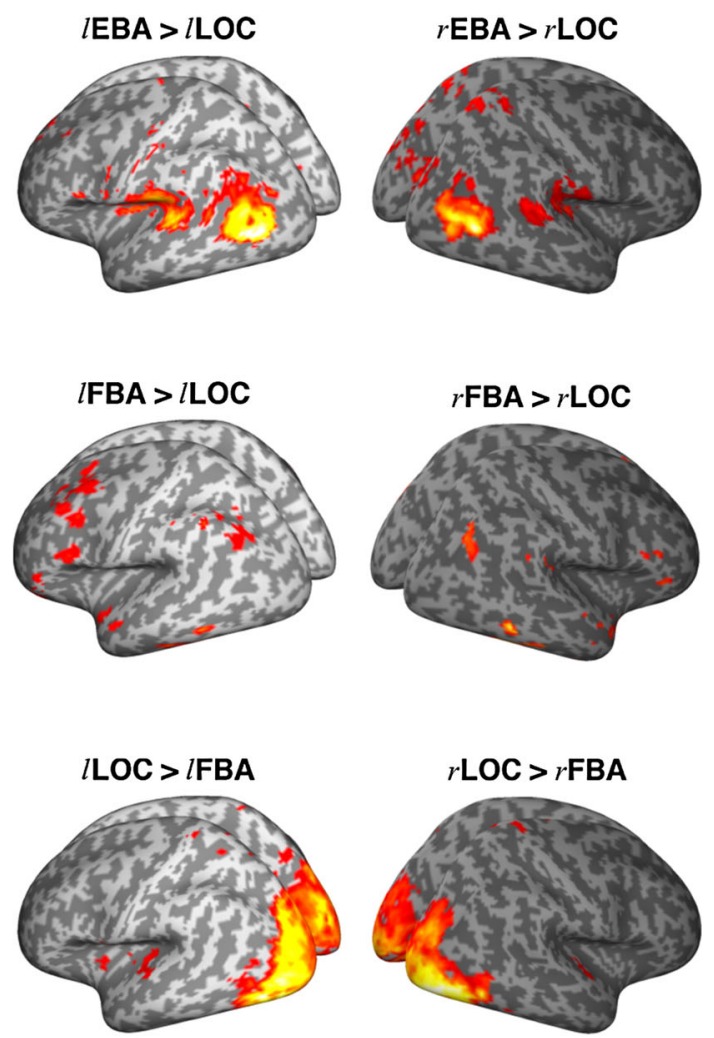

forming threshold of $p<0.001$. Colours (red-yellow) indicate voxelwise $t$ values, for scale see Fig. 2. For a list of covered regions, see supplementary material (Table S1)

characterized by its strong connections with the fusiform gyrus and the inferior temporal lobe. The connectivity fingerprint of LOC is less biased towards connections with either dorsal or ventral stream regions. Finally, only EBA and LOC, but not FBA, connect strongly with primary visual areas. When testing for specialization between hemispheric homologues of these regions, the connectivity fingerprints emphasized differences between left and right EBA $(p=0.03)$, but not FBA $(p=0.19)$ and LOC $(p=0.18)$. Logistic regression revealed that the hemispheric specialization of EBA was mostly driven by stronger connectivity of right than left EBA with OP $(p=0.04)$, STS $(p=0.01)$, and FFG $(p=0.04)$, but notably not by BA5 $(p=0.98)$, BA7a $(p=0.69)$, and BA2 $(p=0.44)$. This suggests that both left and right EBA were strongly connected with superior parietal regions, but differed in their coupling with temporal regions.

\section{Part II: hypothesis-driven analyses}

ROI-based functional connectivity After characterizing the connectivity fingerprints, we directly tested whether EBA would show stronger connectivity strength with the dorsal stream and is more likely to resemble a dorsalstream connectivity profile, as compared to FBA and LOC 

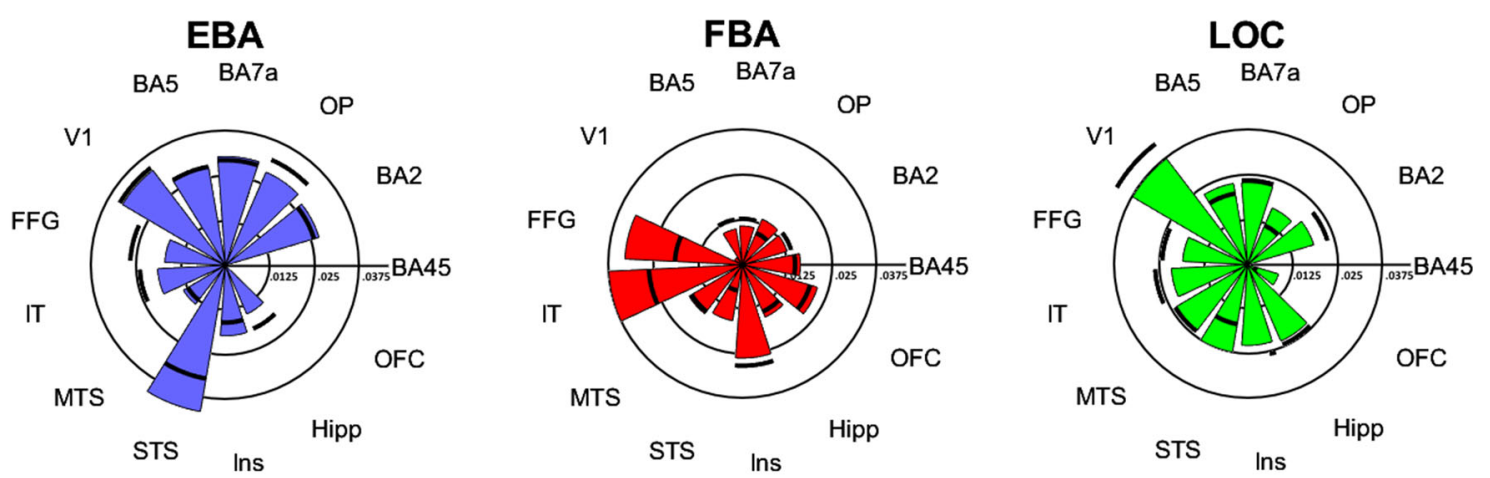

Fig. 4 Connectivity fingerprints of seed regions EBA, FBA, and LOC for left (wedges) and right (lines) hemispheres. Only positive connections are shown. The scale indicates beta values. $B A$

Brodmann area, $O P$ operculum, STS superior temporal sulcus, MTS medial temporal sulcus, $I T$ inferior temporal sulcus, $F F G$ fusiform gyrus, $O F C$ orbito-frontal cortex, Hipp hippocampus, Ins insula

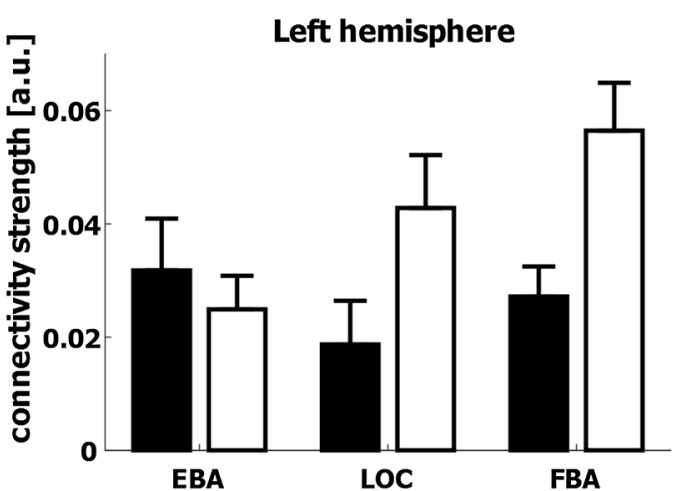

Fig. 5 Resting-state connectivity strength (beta values) between seed regions (EBA, FBA, and LOC) and target regions in the dorsal visual stream (in black) or in the ventral visual stream (in white). In both hemispheres, EBA shows relatively stronger connectivity to the

(Fig. 5). First, we assessed the connectivity strength of EBA, FBA, and LOC with the dorsal and ventral streams. Using large ROIs spanning ventral and dorsal-stream regions based on the whole-brain cortical parcellation atlas (HCP-MMP1.0, Glasser et al. 2016; see "Methods" for details), a $3 \times 2$ ANOVA with factors seeds (EBA, FBA, and $\mathrm{LOC}$ ) and target regions (dorsal and ventral) indicated a significant interaction between seed and target regions in terms of resting-state connectivity strength $[F(2,60)=13.03, p<0.001]$, while the three-way interaction including hemisphere (left and right), seed (EBA, FBA, and LOC), and target region (dorsal and ventral) was not significant $[F(2,60)=2.65, p=0.079]$. Accordingly, the seed-target interaction was observed for both left hemisphere $[F(2,60)=7.92, p=0.002]$ and right hemisphere $[F(2,60)=12.23, \quad p<0.001 ; \quad$ all Bonferroni corrected].

The underlying $2 \times 2$ interaction with seed regions EBA and LOC was significant $[F(1,30)=14.68$, $p=0.003]$. The $2 \times 2$ interaction with seed regions EBA

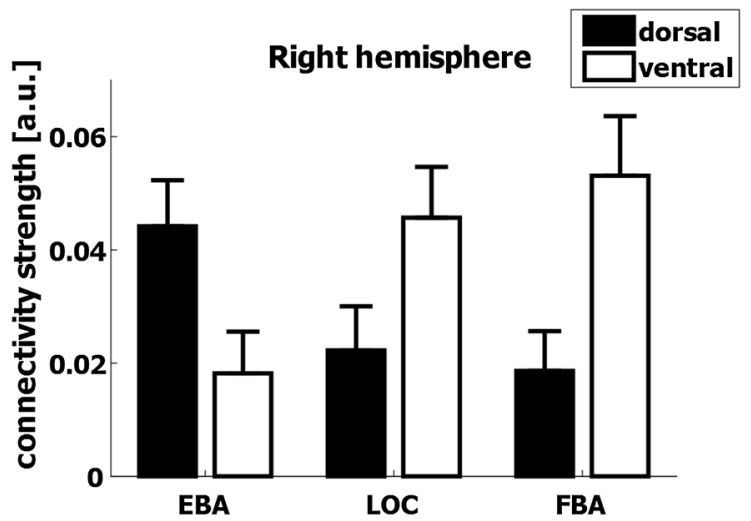

dorsal stream compared to LOC and FBA, which both show relatively stronger connectivity with the ventral stream. Error bars indicate the standard error of the mean

and FBA was also significant $[F(1,30)=25.87$, $p<0.001]$. The interaction between seed regions LOC and FBA and target regions (dorsal and ventral) was not significant $[F(1,30)=0.61, p>0.10$; all Bonferroni corrected]. The same patterns were observed for both hemispheres. As shown in Fig. 5, EBA shows relatively stronger connectivity to the dorsal target ROI compared to LOC and FBA, which both show relatively stronger connectivity with the ventral stream ROI. Whereas LOC and FBA show greater connectivity strength to ventral stream ROIs [LOC: $F(1,30)=5.03, \quad p=0.032 ; \quad$ FBA: $F(1,30)=10.74, p=0.003]$, the difference for EBA between dorsal and ventral target regions was only a trend towards stronger connectivity with the dorsal-stream ROI $[F(1,30)=3.24, p=0.082]$.

A detailed analysis of the connectivity strength between the seed regions and all regions of the HCP-MMP1.0 atlas individually shows that these effects are consistent over a large number of regions and are not driven by any outliers (Supplementary material S3). This analysis shows that the 
findings presented in Fig. 5 are robust across the whole of the lateral parietal and inferior temporal cortex and do not depend on minor and arbitrary changes to our definition of dorsal and ventral stream regions of interest.

Seed-region classification Next, complementary to the analyses of connectivity strength, we aimed to classify whole-brain connectivity patterns of the seed regions (EBA, FBA, and LOC) as either 'dorsal' or 'ventral'. We based the classification on the resemblance of the seeds' whole-brain connectivity patterns to those of the segmented regions from the HCP-MMP1.0 atlas (Glasser et al. 2016) used to define dorsal and ventral streams (see Definition of dorsal and ventral stream target regions; Table 2). Figure 6 shows the average classification outcome for different classifiers (i.e., different numbers of neighbours $k$ in a $k$-nearest-neighbour classification, see "Methods"). However, $k$ was included as factor in the analyses. Classification outcome differed between seed regions for both hemispheres [left: $\chi^{2}(2,558)=79.93, p<0.001$; right: $\chi^{2}(2,558)=104.42, p<0.001$; Friedman's non-parametric test], with a significant interaction between seed region and hemisphere $[F(2,360)=14.70, p<0.001]$. In the left hemisphere, EBA was more likely to be classified as dorsal compared to both $\operatorname{LOC}\left[\chi^{2}(1,372)=24.29, p<0.001\right]$ and FBA $\left[\chi^{2}(1,372)=79.09, p<0.001\right]$, and LOC was more

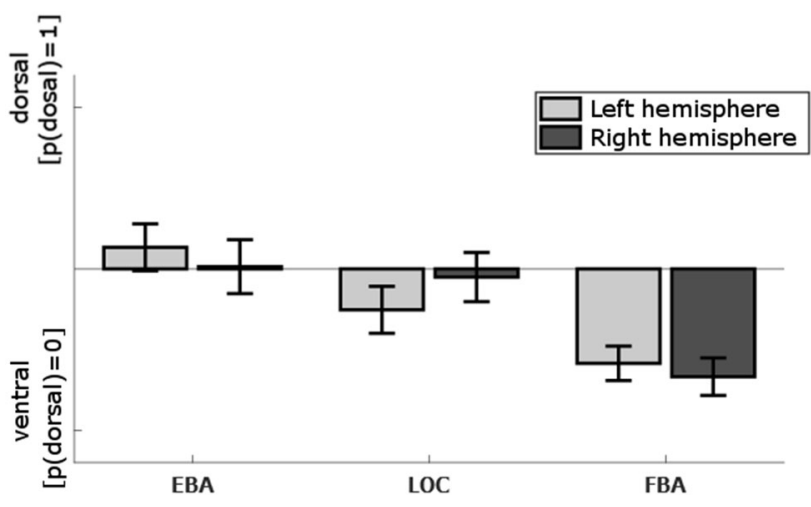

Fig. 6 Classification result of seed regions EBA, LOC, and FBA classified as either 'dorsal' or 'ventral' based on whole-brain connectivity patterns of several dorsal and ventral stream areas (see Table 2; Definition of dorsal and ventral stream target regions) for left and right hemispheres. Seed regions differ in classification outcome: EBA is consistently classified more dorsal than FBA, with LOC between EBA and FBA. Error bars indicate the standard error of the mean. Bars represent the probability of a seed region being classified as 'dorsal', ranging from 0 (always ventral) to 1 (always dorsal). Bars are centred to $p=0.5$, i.e., equally likely to be classified as dorsal or ventral. Presented classification outcomes are based on average classification outcomes over different classifier parameters (i.e., $k$ in a $k$-nearest-neighbours classification). Full results for different $k$ values are presented in the supplementary material (Figure S2) likely classified as dorsal than FBA $\left[\chi^{2}(1,372)=23.73\right.$, $p<0.001]$. In the right hemisphere, there was no difference in classification outcome between EBA and LOC ( $p>0.10$ ), but both EBA and LOC were more likely to be classified as dorsal than FBA [EBA vs. FBA: $\chi^{2}(1,372)=100.83, \quad p<0.001 ; \quad$ LOC vs. FBA: $\left.\chi^{2}(1,372)=77.29, p<0.001\right]$. Interestingly, classification outcome depended on the level of $k$, as was shown in a number of interactions with $k$ [hemisphere $\times$ seed region $\times k: \quad F(12,360)=4.24, \quad p<0.001 ; \quad$ seed region $\times k: \quad F(12,360)=12.36, \quad p<0.001$; hemisphere $\times k: F(6,360)=4.06, p=0.001$; and an effect of $k: F(6,360)=2.58, p=0.020]$. Visual inspection of the data revealed that with small $k(k<5)$, all seed regions are classified predominantly ventral or neutral, whereas classification outcome for EBA changes towards dorsal with larger $k(4<k<9)$. Importantly, the relationship between the regions of interest is independent of $k$, with the dorsal classification of EBA $>$ LOC $>$ FBA in the left hemisphere and $\mathrm{EBA} \cong \mathrm{LOC}>\mathrm{FBA}$ in the right hemisphere. Classification outcome for FBA is consistently ventral, irrespective of $k$. Detailed results are presented in the supplementary material (Figure S2).

Probabilistic tractography of diffusion-weighted MRI Finally, we aimed to complement and confirm the resting-state fMRI approach using probabilistic tractography on diffusion-weighted MRI data. Tractography showed that all three seed regions had more projections to ventral than dorsal regions in absolute terms. This is expected given their spatial proximity to the ventral target. However, it also revealed that the relative distribution of ventral and dorsal projections differed per region: EBA was characterized by strong dorsal projections and FBA by strong ventral projections, with an intermediate pattern for LOC (Fig. 7). Specifically, we found significant interactions of seed by target region in connection strength (log-transformed tract probability) in both hemispheres [left: $F(2,62)=55.32, \quad p<0.001 ; \quad$ right: $\quad F(2,62)=21.61$, $p<0.001]$. This index differed between hemispheres, as indicated by the significant three-way interaction between seed region, target region, and hemisphere $[F(2,62)=4.00, p=0.023]$, likely caused by hemispheric differences of FBA as shown by a significant interaction with factors hemisphere and target region for FBA $[F(1,31)=6.65, p=0.015]$, which was not significant for EBA and LOC (both $p>0.10$ ). Post-hoc tests revealed that seed regions differed significantly in terms of their probability of connecting to the dorsal-stream ROIs in both hemispheres [left: $F(2,62)=36.82, \quad p<0.001$; right: $F(2,62)=16.81, p<0.001]$. Specifically, EBA's probability of connecting to the dorsal stream was stronger than 


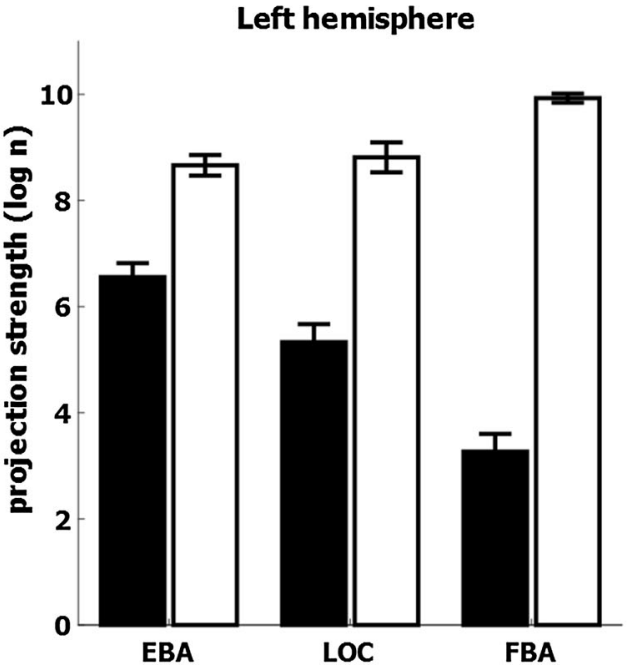

Fig. 7 Connectivity strength of EBA, FBA, and LOC with ventral and dorsal regions indexed using probabilistic tractography of diffusion-weighted MRI. All three seed regions show more projections to ventral than dorsal regions, but the relative distribution

LOC's and FBA's [left: $t(31)=3.42, \quad p=0.006$; $t(31)=8.98, p<0.001$; right: $t(31)=6.58, p<0.001$; $t(31)=5.06, p<0.001]$. There was a significant difference between LOC and FBA in the left hemisphere, but not in the right hemisphere [left: $t(31)=4.76, p<0.001$; right: $t(31)=1.49, p>0.10$; all Bonferroni corrected]. Similarly, seed regions differed significantly in terms of their probability of connecting to the ventral stream ROIs in both hemispheres [left: $F(2,62)=14.67, p<0.001$; right: $F(2,62)=12.80, p<0.001]$. Specifically, in both hemispheres, FBA had a stronger probability of connecting to ventral stream than EBA and LOC [left: $t(31)=6.17$, $p<0.001 ; t(31)=4.06, p=0.001$; right: $t(31)=4.45$, $p<0.001 ; t(31)=4.58, p<0.001]$. EBA and LOC did not differ significantly in either hemisphere (all $p>0.10$, all Bonferroni corrected). Although FBA is closer in distance to ventral stream regions than EBA and LOC, these effects cannot simply be explained by a bias of distance between the seed and the target regions: all seed regions are similarly distant from dorsal-stream regions and EBA and LOC are equidistant from inferior temporal, yet a striking difference in connectivity probability was observed. The probabilistic tractography analysis confirms the rs-fMRI finding, indicating that EBA is relatively strongly connected with the dorsal stream.

A detailed analysis of the connection strength between the seed regions and all regions of the HCP-MMP1.0 atlas individually shows that these effects are consistent over a large number of regions and are not driven by any outliers (Supplementary material S4), showing that the findings presented in Fig. 7 are robust across the whole of the lateral parietal and inferior temporal cortex.

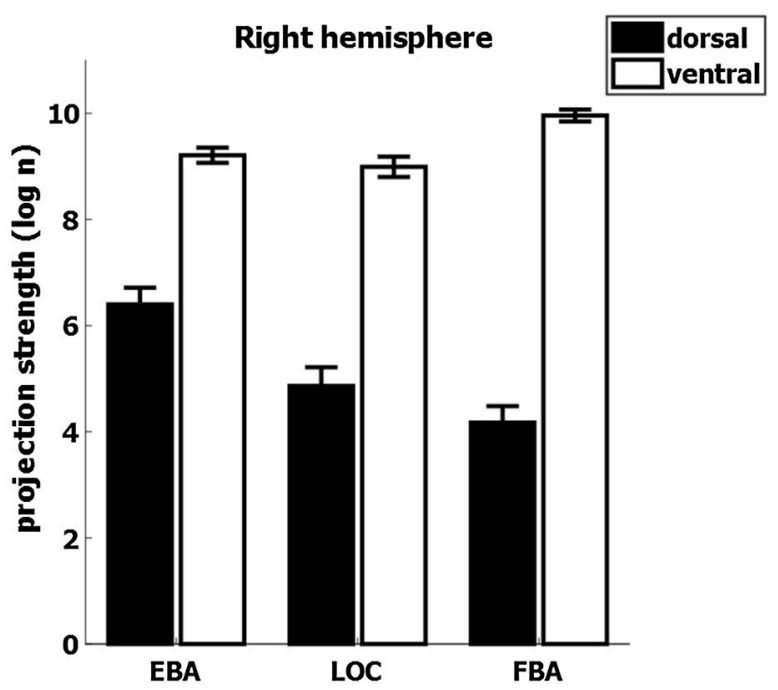

differed: EBA is characterized by relatively stronger dorsal projections and FBA by relatively stronger ventral projections. Error bars indicate the standard error of the mean

\section{Discussion}

This study tests whether EBA has stronger connectivity with the dorsal visuomotor stream than two nearby perceptual areas in the occipito-temporal cortex, LOC and FBA. The main finding of this study is that EBA is more strongly connected to parietal regions than FBA and LOC, both functionally and structurally. This observation clarifies the ongoing debate on EBA function (Downing and Peelen 2011, 2016), providing anatomical evidence for the notion that EBA is more closely engaged with portions of the parietal cortex than other occipito-temporal areas. EBA's connectivity profile supports the suggestion that its contributions to goal-oriented behaviour are different from those of other portions of the ventral visual stream (Kühn et al. 2011; van Nuenen et al. 2012; Zimmermann et al. 2012, 2016).

\section{Dorsal-stream affinity of the extrastriate body area}

The dw-MRI tractography index of structural connectivity reflected the proximity and connectivity of all investigated occipito-temporal regions to the ventral stream. In addition, it revealed that EBA's affinity with the dorsal stream is markedly stronger than that of LOC and FBA (Fig. 7), revealing a more extensive connectivity profile than previously identified (Beer et al. 2013). This effect was present for both the left and right hemispheric homologues. The resting-state functional coupling of EBA also revealed a greater affinity, both in strength and profile, with the dorsal stream than with the ventral stream (Fig. 5). At a whole-brain level, EBA has strong functional connections 
with occipital regions. In addition, EBA is functionally coupled with the superior parietal lobe, the parietal operculum, and the postcentral gyrus (Fig. 2), in line with previous studies showing that EBA is connected to regions involved in various action related processes (e.g., Beer et al. 2013; Zimmermann et al. 2013; Orgs et al. 2016; Simos et al. 2017). These parietal regions are involved in somatosensory processing (Dijkerman and de Haan 2007), in the integration of somatosensory and visual information during reaching/grasping movements (Fogassi and Luppino 2005), and in the estimation of future body states during action execution (Wolpert et al. 1998). Those parietal regions could provide EBA with access to somatosensory representations of one's own current body posture. This functional connectivity profile fits with the observation that EBA is sensitive to discrepancies between one's own current body posture and predicted body postures (Arzy et al. 2006; Zimmermann et al. 2012, 2013; Limanowski et al. 2014; Limanowski and Blankenburg 2016). It remains unclear whether the whole EBA or only a portion has access to both visual and somatosensory information, similar to how the lateral occipital complex contains a subsection sensitive to both visual and tactile object information (Amedi et al. 2001). Future investigations could test whether EBA could be further subdivided in modality-related integrative units, as other parts of the lateral occipitotemporal cortex (see Bracci et al. 2012).

The exceptionally strong connectivity of EBA with postcentral, opercular, and dorsomedial portions of the parietal cortex does not fit with a purely visuo-perceptual role for this area. Access to somatosensory information would be irrelevant for an EBA devoted to identify visually presented body stimuli, or to process the perceptual consequences of executed motor acts (Downing et al. 2001; Downing and Peelen 2011). In contrast, knowing the current postural configuration of one's own body is crucial for an EBA involved in motor control (van Nuenen et al. 2012; Zimmermann et al. 2012, 2016). EBA's access to both visual and haptic information (Kitada et al. 2009, 2014) supports the notion that this region biases the sensorimotor transformations implemented in the parieto-frontal circuits with a postural goal-state derived from learned knowledge (Verhagen et al. 2012).

\section{EBA and FBA}

FBA, which is consistently co-activated with EBA (Peelen and Downing 2005; Weiner and Grill-Spector 2010), shows weaker connectivity than EBA with dorsal-stream regions. This could suggest that FBA plays primarily a perceptual role, namely, the role originally proposed for EBA
(Downing et al. 2001). Accordingly, FBA and EBA have been proposed to be hierarchically organized regions involved in body perception with FBA representing more holistic, whole-body information than EBA, as well as dynamic movements and identity (Hodzic et al. 2009; Ewbank et al. 2011; Orgs et al. 2016). Alternatively, the FBA connectivity isolated in this study raises the possibility that this region is also involved in providing desired goal states to action plans, although through a different circuit than EBA. Namely, FBA connectivity with areas 45, 46, and IFS could mediate perceptual influences on motor planning through prefrontal cortex, such as the selection of action targets and objects according to abstract goals (Milner and Goodale 2008). These ventral visual stream influences on action selection would become relevant only during late planning stages of actions (Milner and Goodale 2008), in contrast to the direct and early influences on motor behaviour exerted by EBA (Zimmermann et al. 2016).

\section{EBA and LOC}

LOC is consistently associated with object perception and recognition (Malach et al. 1995; Amedi et al. 2001; GrillSpector et al. 2001; Kourtzi and Kanwisher 2001), alike EBA's involvement in body perception. The similarities extend further, as not only EBA, but also LOC has been associated with action planning (Verhagen et al. 2008; Gallivan et al. 2015). Specifically, LOC is thought to provide perceptual information about objects used for grasping (Verhagen et al. 2008). EBA, in contrast, is thought to represent desired body postures used during planning and execution of goal-directed actions (Zimmermann et al. 2012, 2016). Previously, we have suggested LOC contributes to the initiation of action planning by providing object-based priors (Verhagen et al. 2012). Recently, we have shown that EBA critically shapes the earliest stages of action planning when one's body posture is relevant for the action outcome (Zimmermann et al. 2016). This highlights the possibility that EBA might provide the dorsal stream with perceptual-based action priors, similar to LOC. Importantly, the functional contributions of LOC and EBA to action and perception are reflected in their respective connectivity profiles, highlighting access to both ventral- and dorsal-stream areas (Figs. 2, 4). Connections of EBA with dorsal-stream regions are stronger than those of LOC, putatively reflecting a more direct involvement in motor control for EBA (Figs. 4, 5, 7). Detailed analyses of their connectivity profiles may reveal specific connections along the dorsal stream that correspond to the dissociable contributions these regions may have to action. 


\section{EBA and MT+}

The cortical extent of EBA has been suggested to overlap at least partly with motion area MT+ (Orban et al. 2004; Peelen et al. 2006; Downing et al. 2007; Ferri et al. 2013). It is yet not clear whether this region's sensitivity to static bodies and moving dots, two very different stimuli, is driven by the same or different neuronal populations. The current study was not focused on resolving this issue or on making inferences specific to the portion of EBA that does not overlap with hMT+. Rather, here, we test a long-standing assumption on EBA functionality, namely, that EBA is a canonical category-specific perceptual area with connections correspondingly biased towards the ventral visual stream. The current findings do not support this assumption. Instead, the observed pattern of connectivity opens the possibility that EBA provides an interface between action and perception, similar to the role of MT+ in planning and control of goal-directed actions (Maunsell and van Essen 1983; Ungerleider and Desimone 1986; Lewis and Van Essen 2000).

\section{Interpretational issues}

Resting-state connectivity and diffusion MRI do not provide information about the directionality of connections. The suggestion that brain regions in the occipito-temporal cortex project onto the motor system implies a directed information flow from occipito-temporal to dorsal-stream regions. Similarly, accounts that emphasize how motor information is used to predict sensory consequences of actions would require the same connections, in the opposite direction. Findings from a recent transcranial magnetic stimulation study, on the same participants, complement the current observations with causal information about the direction and the temporal relevance of these connections, showing that EBA influences action planning well before IPS (Zimmermann et al. 2016).

Resting-state connectivity is an indirect measure of anatomy that cannot distinguish between direct and indirect connections (Honey et al. 2009; Passingham 2013). For instance, correlation of BOLD responses between two regions can be caused by a third region that projects to both, in the absence of a direct connection between the two BOLD-correlated regions. Nonetheless, this approach has proven to be very sensitive to differences in correlation patterns between regions, also in the occipito-temporal cortex (Hutchison et al. 2014). Diffusion MRI tractography has different limitations; for instance, its results are strongly biased by the distance between seed- and target regions. Moreover, diffusion tractography methods have trouble distinguishing between crossing and curved fibres within a voxel, leading to a sub-optimal balance between sensitivity and specificity (Thomas et al. 2014). Although diffusion tractography has been repeatedly proven to closely match the golden-standard of tracing results (Croxson et al. 2005; Dauguet et al. 2007; Jbabdi et al. 2013; Azadbakht et al. 2015), the goal of dw-MRI tractography in this study is dissimilar from that of tracing studies. We aim to quantify the probability of regions being part of the same connectional system, and markedly do not aim to qualify the presence of single-synapse connections between these regions. Furthermore, the current analyses are designed to complement rs-fMRI and dw-MRI strengths while minimizing their weaknesses. For instance, we have used subject-specific localised seed regions and a priori target regions, to balance specificity and sensitivity. Moreover, in line with most diffusion tractography studies, we do not draw inferences on absolute connectivity probabilities, but compare relative values across regions with similar distances.

Some analyses resulted in hemispheric differences, suggesting that left and right hemispheric regions may have different specializations, consistent with suggestions of hemispheric specialization in motor control and perception (Schluter et al. 1998; Downing et al. 2001; de Lange et al. 2005; Peelen and Downing 2005; Arzy et al. 2006; Willems et al. 2009; Zimmermann et al. 2012; Limanowski et al. 2014). However, in this study, no clear patterns were observed with respect to hemispheric differences and findings of hemispheric differences were not consistent over complementary analyses. Future studies are required to investigate whether and how structural and functional connectivity of the investigated regions differ between hemispheres.

In this study we focus on the motor aspects of the dorsal stream, but it is worth noting that this circuit is also involved in visuospatial perception (e.g., Ungerleider and Haxby 1994). It is conceivable that differences in dorsalstream connectivity of EBA, LOC, and FBA reflect differences in the regions' contributions to visuospatial perception. Namely, one could speculate that recognition of body parts could contribute both to the estimation of the relative position of other persons and their posture to oneself (putatively mediated by EBA) and to the identification of the owner of the body part (putatively mediated by FBA based on holistic body representations; Taylor et al. 2007). In this framework, LOC would be expected to show an intermediate connectivity profile, given that relative position and identity are often both highly relevant for objects. Along these lines, regions computing identification per se, such as FFA processing face information, are, therefore, expected to have a more ventral connectivity profile. Conversely, this framework also predicts that the parahippocampal place area (PPA) has a comparably dorsally oriented connectivity profile, as the relative spatial 
location of places is often relevant in addition to the identification of these places.

\section{Conclusions}

Here, we provide anatomical evidence that the extrastriate body area has strong interactions with parietal cortex, allowing it to exchange information with dorsal-stream areas. Diffusion tractography confirmed the relative dorsalstream affinity of EBA. Contrasting with EBA, the fusiform body area could be robustly classified as a ventral region based on its connectivity profile. It is characterized by strong connections to higher perceptual areas in the inferior temporal cortex and a lack of marked connections to posterior parietal and postcentral regions involved in motor control. The lateral occipital complex revealed an intermediate pattern with varying affinity for ventral and dorsal visual streams, suggesting that it might not only serve as a gateway for ventral, but also for dorsal-stream processing. These observations provide an anatomical ground for the suggestion that EBA is not only involved in body perception (Downing et al. 2001; Downing and Peelen 2011), but also contributes to action planning by anticipating body states (Zimmermann et al. 2012, 2016).

Taken together, this study adds to a growing body of the literature suggesting that the boundary between dorsal and ventral visual processing streams is not as clear as it was suggested initially (Goodale and Milner 1992). In fact, studies reporting existence of several parallel processing streams (Kravitz et al. 2011, 2013) and observations that regions are well connected to both streams, such as those reported here (see also Orgs et al. 2016), challenge this initial view (see also Milner 2017).

Acknowledgements This research was supported by VICI Grant 453-08-002 from the Netherlands Organisation for Scientific Research (NWO), awarded to IT. The work of RBM is supported by NWO (452-13-015) and the BBSRC UK (BB/N019814/1). FPdL is supported by Grants from NWO (452-13-016), the James S McDonnell Foundation (Understanding Human Cognition, 220020373) and the European Union Horizon 2020 Program (ERC Starting Grant 678286, "Contextvision"). The work of LV is supported by the Marie Curie Intra-European Fellowship within the European Union's 7th Framework Program FP7/2007-2013; MC-IEF623513 and the Royal Netherlands Academy of Arts and Sciences (KNAW) (Prijs Akademiehoogleraar 2012 to Peter Hagoort, supporting LV).

\section{Compliance with ethical standards}

Statement of human rights All procedures performed in studies involving human participants were in accordance with the ethical standards of the institutional and/or national research committee and with the 1964 Helsinki declaration and its later amendments or comparable ethical standards. All participants gave written informed consent according to the institutional guidelines of the local ethics committee (CMO region Arnhem-Nijmegen, The Netherlands).

Open Access This article is distributed under the terms of the Creative Commons Attribution 4.0 International License (http://crea tivecommons.org/licenses/by/4.0/), which permits unrestricted use, distribution, and reproduction in any medium, provided you give appropriate credit to the original author(s) and the source, provide a link to the Creative Commons license, and indicate if changes were made.

\section{References}

Amedi A, Malach R, Hendler T, Peled S, Zohary E (2001) Visuohaptic object-related activation in the ventral visual pathway. Nat Neurosci 4:324-330

Arzy S, Thut G, Mohr C, Michel CM, Blanke O (2006) Neural basis of embodiment: distinct contributions of temporoparietal junction and extrastriate body area. J Neurosci 26:8074-8081

Ashburner J, Friston KJ (1999) Nonlinear spatial normalization using basis functions. Hum Brain Mapp 7:254-266

Astafiev SV, Stanley CM, Shulman GL, Corbetta M (2004) Extrastriate body area in human occipital cortex responds to the performance of motor actions. Nat Neurosci 7:542-548

Azadbakht H, Parkes LM, Haroon HA, Augath M, Logothetis NK, de Crespigny A, D'Arceuil HE, Parker GJM (2015) Validation of high-resolution tractography against in vivo tracing in the macaque visual cortex. Cereb Cortex 25:4299-4309

Beer AL, Plank T, Meyer G, Greenlee MW (2013) Combined diffusion-weighted and functional magnetic resonance imaging reveals a temporal-occipital network involved in auditory-visual object processing. Front Integr Neurosci 7:5

Behrens TEJ, Berg HJ, Jbabdi S, Rushworth MFS, Woolrich MW (2007) Probabilistic diffusion tractography with multiple fibre orientations: what can we gain? Neuroimage 34:144-155

Biswal B, Yetkin FZ, Haughton VM, Hyde JS (1995) Functional connectivity in the motor cortex of resting human brain using echo-planar MRI. Magn Reson Med 34:537-541

Bracci S, Cavina-Pratesi C, Ietswaart M, Caramazza A, Peelen MV (2012) Closely overlapping responses to tools and hands in left lateral occipitotemporal cortex. J Neurophysiol 107:1443-1456

Brett M, Anton JL, Valabregue R, Poline JB (2002) Region of interest analysis using an SPM toolbox [WWW Document]. Neuroimage. http://marsbar.sourceforge.net/about.html

Croxson PL, Johansen-Berg H, Behrens TEJ, Robson MD, Pinsk MA, Gross CG, Richter W, Richter MC, Kastner S, Rushworth MFS (2005) Quantitative investigation of connections of the prefrontal cortex in the human and macaque using probabilistic diffusion tractography. J Neurosci 25:8854-8866

Dauguet J, Peled S, Berezovskii V, Delzescaux T, Warfield SK, Born R, Westin C-F (2007) Comparison of fiber tracts derived from in vivo DTI tractography with $3 \mathrm{D}$ histological neural tract tracer reconstruction on a macaque brain. Neuroimage 37:530-538

David N, Cohen MX, Newen A, Bewernick BH, Shah NJ, Fink GR, Vogeley K (2007) The extrastriate cortex distinguishes between the consequences of one's own and others' behavior. Neuroimage 36:1004-1014

de Lange FP, Hagoort P, Toni I (2005) Neural topography and content of movement representations. J Cogn Neurosci 17:97-112

Dijkerman HC, de Haan EHF (2007) Somatosensory processes subserving perception and action. Behav Brain Sci 30:189

Downing PE, Peelen MV (2011) The role of occipitotemporal bodyselective regions in person perception. Cogn Neurosci 2:186-203 
Downing PE, Peelen MV (2016) Body selectivity in occipitotemporal cortex: causal evidence. Neuropsychologia 83:138-148

Downing PE, Jiang Y, Shuman M, Kanwisher N (2001) A cortical area selective for visual processing of the human body. Science 293:2470-2473

Downing PE, Wiggett AJ, Peelen MV (2007) Functional magnetic resonance imaging investigation of overlapping lateral occipitotemporal activation using multi-voxel pattern analysis. J Neurosci 27:226-233

Eickhoff SB, Stephan KE, Mohlberg H, Grefkes C, Fink GR, Amunts $\mathrm{K}$, Zilles K (2005) A new SPM toolbox for combining probabilistic cytoarchitectonic maps and functional imaging data. Neuroimage 25:1325-1335

Ewbank MP, Lawson RP, Henson RN, Rowe JB, Passamonti L, Calder AJ (2011) Changes in "top-down" connectivity underlie repetition suppression in the ventral visual pathway. J Neurosci 31:5635-5642

Felleman DJ, Van Essen DC (1991) Distributed hierarchical processing in the primate cerebral cortex. Cereb Cortex 1:1-47

Ferri S, Kolster H, Jastorff J, Orban GA (2013) The overlap of the EBA and the MT/V5 cluster. Neuroimage 66:412-425

Fogassi L, Luppino G (2005) Motor functions of the parietal lobe. Curr Opin Neurobiol 15:626-631

Fox MD, Raichle ME (2007) Spontaneous fluctuations in brain activity observed with functional magnetic resonance imaging. Nat Rev Neurosci 8:700-711

Friston KJ, Ashburner J, Frith CD, Poline J-B, Heather JD, Frackowiak RSJ (1995a) Spatial registration and normalization of images. Hum Brain Mapp 3:165-189

Friston KJ, Holmes AP, Poline JB, Grasby PJ, Williams SC, Frackowiak RS, Turner R (1995b) Analysis of fMRI time-series revisited. Neuroimage 2:45-53

Gallivan JP, McLean DA, Valyear KF, Pettypiece CE, Culham JC (2011) Decoding action intentions from preparatory brain activity in human parieto-frontal networks. $\mathbf{J}$ Neurosci 31:9599-9610

Gallivan JP, Johnsrude IS, Randall Flanagan J (2015) Planning ahead: object-directed sequential actions decoded from human frontoparietal and occipitotemporal networks. Cereb Cortex 26:708-730

Glasser MF, Sotiropoulos SN, Wilson JA, Coalson TS, Fischl B, Andersson JL, Xu J, Jbabdi S, Webster M, Polimeni JR, Van Essen DC, Jenkinson M, WU-Minn HCP Consortium (2013) The minimal preprocessing pipelines for the Human Connectome Project. Neuroimage. 80:105-124

Glasser MF, Coalson TS, Robinson EC, Hacker CD, Harwell J, Yacoub E, Ugurbil K, Andersson J, Beckmann CF, Jenkinson M, Smith SM, Van Essen DC (2016) A multi-modal parcellation of human cerebral cortex. Nature 536:171-178

Goodale MA, Milner AD (1992) Separate visual pathways for perception and action. Trends Neurosci 15:20-25

Grill-Spector K, Kourtzi Z, Kanwisher N (2001) The lateral occipital complex and its role in object recognition. Vision Res 41:1409-1422

Hagmann P, Cammoun L, Gigandet X, Meuli R, Honey CJ, Wedeen VJ, Sporns O (2008) Mapping the structural core of human cerebral cortex. PLoS Biol 6:e159

Hodzic A, Kaas A, Muckli L, Stirn A, Singer W (2009) Distinct cortical networks for the detection and identification of human body. Neuroimage 45:1264-1271

Honey CJ, Sporns O, Cammoun L, Gigandet X, Thiran JP, Meuli R, Hagmann P (2009) Predicting human resting-state functional connectivity from structural connectivity. Proc Natl Acad Sci USA 106:2035-2040

Hutchison RM, Culham JC, Everling S, Flanagan JR, Gallivan JP (2014) Distinct and distributed functional connectivity patterns across cortex reflect the domain-specific constraints of object, face, scene, body, and tool category-selective modules in the ventral visual pathway. Neuroimage 96:216-236

James TW, Culham J, Humphrey GK, Milner AD, Goodale MA (2003) Ventral occipital lesions impair object recognition but not object-directed grasping: an fMRI study. Brain 126:2463-2475

Jbabdi S, Lehman JF, Haber SN, Behrens TE (2013) Human and monkey ventral prefrontal fibers use the same organizational principles to reach their targets: tracing versus tractography. J Neurosci 33:3190-3201

Kanwisher N, McDermott J, Chun MM (1997) The fusiform face area: a module in human extrastriate cortex specialized for face perception. J Neurosci 17:4302-4311

Kitada R, Johnsrude IS, Kochiyama T, Lederman SJ (2009) Functional specialization and convergence in the occipitotemporal cortex supporting haptic and visual identification of human faces and body parts: an fMRI study. J Cogn Neurosci 21:2027-2045

Kitada R, Yoshihara K, Sasaki AT, Hashiguchi M, Kochiyama T, Sadato N (2014) The brain network underlying the recognition of hand gestures in the blind: the supramodal role of the extrastriate body area. J Neurosci 34:10096-10108

Kourtzi Z, Kanwisher N (2001) Representation of perceived object shape by the human lateral occipital complex. Science 80:293

Kravitz DJ, Saleem KS, Baker CI, Mishkin M (2011) A new neural framework for visuospatial processing. Nat Rev Neurosci $12: 217-230$

Kravitz DJ, Saleem KS, Baker CI, Ungerleider LG, Mishkin M (2013) The ventral visual pathway: an expanded neural framework for the processing of object quality. Trends Cogn Sci 17:26-49

Kühn S, Keizer A, Rombouts SARB, Hommel B (2011) The functional and neural mechanism of action preparation: roles of EBA and FFA in voluntary action control. J Cogn Neurosci 23:214-220

Lewis JW, Van Essen DC (2000) Corticocortical connections of visual, sensorimotor, and multimodal processing areas in the parietal lobe of the macaque monkey. J Comp Neurol 428:112-137

Limanowski J, Blankenburg F (2016) Integration of visual and proprioceptive limb position information in human posterior parietal, premotor, and extrastriate cortex. J Neurosci 36:2582-2589

Limanowski J, Lutti A, Blankenburg F (2014) The extrastriate body area is involved in illusory limb ownership. Neuroimage $86: 514-524$

Lingnau A, Downing PE (2015) The lateral occipitotemporal cortex in action. Trends Cogn Sci 19:268-277

Malach R, Reppas JB, Benson RR, Kwong KK, Jiang H, Kennedy WA, Ledden PJ, Brady TJ, Rosen BR, Tootell RB (1995) Object-related activity revealed by functional magnetic resonance imaging in human occipital cortex. Proc Natl Acad Sci USA 92:8135-8139

Mars RB, Sallet J, Schuffelgen U, Jbabdi S, Toni I, Rushworth MFS (2011) Connectivity-based subdivisions of the human right "temporoparietal junction area": evidence for different areas participating in different cortical networks. Cereb Cortex 22:1894-1903

Mars RB, Sallet J, Neubert F-X, Rushworth MFS (2013) Connectivity profiles reveal the relationship between brain areas for social cognition in human and monkey temporoparietal cortex. Proc Natl Acad Sci 110:10806-10811

Mars RB, Foxley S, Verhagen L, Jbabdi S, Sallet J, Noonan MP, Neubert F-X, Andersson JL, Croxson PL, Dunbar RIM, Khrapitchev AA, Sibson NR, Miller KL, Rushworth MFS (2016a) The extreme capsule fiber complex in humans and macaque monkeys: a comparative diffusion MRI tractography study. Brain Struct Funct 221:4059-4071 
Mars RB, Verhagen L, Gladwin TE, Neubert F-X, Sallet J, Rushworth MFS (2016b) Comparing brains by matching connectivity profiles. Neurosci Biobehav Rev 60:90-97

Maunsell JH, van Essen DC (1983) The connections of the middle temporal visual area (MT) and their relationship to a cortical hierarchy in the macaque monkey. J Neurosci 3:2563-2586

Milner AD (2017) How do the two visual streams interact with each other? Exp Brain Res 235:1297-1308

Milner AD, Goodale MA (2008) Two visual systems re-viewed. Neuropsychologia 46:774-785

Mishkin M, Ungerleider LG (1982) Contribution of striate inputs to the visuospatial functions of parieto-preoccipital cortex in monkeys. Behav Brain Res 6:57-77

Neubert F-X, Mars R, Thomas A, Sallet J, Rushworth MS (2014) Comparison of human ventral frontal cortex areas for cognitive control and language with areas in monkey frontal cortex. Neuron 81:700-713

Nichols TE, Holmes AP (2002) Nonparametric permutation tests for functional neuroimaging: a primer with examples. Hum Brain Mapp 15:1-25

O'Reilly JX, Croxson PL, Jbabdi S, Sallet J, Noonan MP, Mars RB, Browning PGF, Wilson CRE, Mitchell AS, Miller KL, Rushworth MFS, Baxter MG (2013) Causal effect of disconnection lesions on interhemispheric functional connectivity in rhesus monkeys. Proc Natl Acad Sci 110:13982-13987

Orban GA, Van Essen D, Vanduffel W (2004) Comparative mapping of higher visual areas in monkeys and humans. Trends Cogn Sci $8: 315-324$

Orgs G, Dovern A, Hagura N, Haggard P, Fink GR, Weiss PH (2016) Constructing visual perception of body movement with the motor cortex. Cereb Cortex 26:440-449

Osher DE, Saxe RR, Koldewyn K, Gabrieli JDE, Kanwisher N, Saygin ZM (2016) Structural connectivity fingerprints predict cortical selectivity for multiple visual categories across cortex. Cereb Cortex 26:1668-1683

Passingham RE (2013) What we can and cannot tell about the wiring of the human brain. Neuroimage 80:14-17

Peelen MV, Downing PE (2005) Selectivity for the human body in the fusiform gyrus. J Neurophysiol 93:603-608

Peelen MV, Wiggett AJ, Downing PE (2006) Patterns of fMRI activity dissociate overlapping functional brain areas that respond to biological motion. Neuron 49:815-822

Pitcher D, Charles L, Devlin JT, Walsh V, Duchaine B (2009) Triple dissociation of faces, bodies, and objects in extrastriate cortex. Curr Biol 19:319-324

Poser BA, Versluis MJ, Hoogduin JM, Norris DG (2006) BOLD contrast sensitivity enhancement and artifact reduction with multiecho EPI: parallel-acquired inhomogeneity-desensitized fMRI. Magn Reson Med 55:1227-1235

Sallet J, Mars RB, Noonan MP, Neubert F-X, Jbabdi S, O'Reilly JX, Filippini N, Thomas AG, Rushworth MF (2013) The organization of dorsal frontal cortex in humans and macaques. J Neurosci 33:12255-12274

Saygin ZM, Osher DE, Koldewyn K, Reynolds G, Gabrieli JDE, Saxe RR (2012) Anatomical connectivity patterns predict face selectivity in the fusiform gyrus. Nat Neurosci 15:321-327
Schluter ND, Rushworth MF, Passingham RE, Mills KR (1998) Temporary interference in human lateral premotor cortex suggests dominance for the selection of movements. A study using transcranial magnetic stimulation. Brain $121(\mathrm{Pt}$ 5):785-799

Simos PG, Kavroulakis E, Maris T, Papadaki E, Boursianis T, Kalaitzakis G, Savaki HE (2017) Neural foundations of overt and covert actions. Neuroimage 152:482-496

Tavor I, Parker Jones O, Mars RB, Smith SM, Behrens TE, Jbabdi S (2016) Task-free MRI predicts individual differences in brain activity during task performance. Science 352:216-220

Taylor JC, Wiggett AJ, Downing PE (2007) Functional MRI analysis of body and body part representations in the extrastriate and fusiform body areas. J Neurophysiol 98:1626-1633

Thomas C, Ye FQ, Irfanoglu MO, Modi P, Saleem KS, Leopold DA, Pierpaoli C (2014) Anatomical accuracy of brain connections derived from diffusion MRI tractography is inherently limited. Proc Natl Acad Sci 111:16574-16579

Tomasino B, Weiss PH, Fink GR (2012) Imagined tool-use in near and far space modulates the extra-striate body area. Neuropsychologia 50:2467-2476

Ungerleider LG, Desimone R (1986) Cortical connections of visual area MT in the macaque. J Comp Neurol 248:190-222

Ungerleider LG, Haxby JV (1994) "What" and "where" in the human brain. Curr Opin Neurobiol 4:157-165

van Nuenen BFL, Helmich RC, Buenen N, van de Warrenburg BPC, Bloem BR, Toni I (2012) Compensatory activity in the extrastriate body area of Parkinson's disease patients. J Neurosci 32:9546-9553

Verhagen L, Dijkerman HC, Grol MJ, Toni I (2008) Perceptuo-motor interactions during prehension movements. J Neurosci 28:4726-4735

Verhagen L, Dijkerman HC, Medendorp WP, Toni I (2012) Cortical dynamics of sensorimotor integration during grasp planning. J Neurosci 32:4508-4519

Weiner KS, Grill-Spector K (2010) Sparsely-distributed organization of face and limb activations in human ventral temporal cortex. Neuroimage 52:1559-1573

Willems RM, Peelen MV, Hagoort P (2009) Cerebral lateralization of face-selective and body-selective visual areas depends on handedness. Cereb Cortex 20:1719-1725

Wolpert DM, Goodbody SJ, Husain M (1998) Maintaining internal representations: the role of the human superior parietal lobe. Nat Neurosci 1:529-533

Young MP (1992) Objective analysis of the topological organization of the primate cortical visual system. Nature 358:152-155

Zimmermann M, Meulenbroek RGJ, de Lange FP (2012) Motor planning is facilitated by adopting an action's goal posture: an fMRI study. Cereb Cortex 22:122-131

Zimmermann M, Toni I, de Lange FP (2013) Body posture modulates action perception. J Neurosci 33:5930-5938

Zimmermann M, Verhagen L, de Lange FP, Toni I (2016) The extrastriate body area computes desired goal states during action planning. eNeuro. doi:10.1523/ENEURO.0020-16.2016 